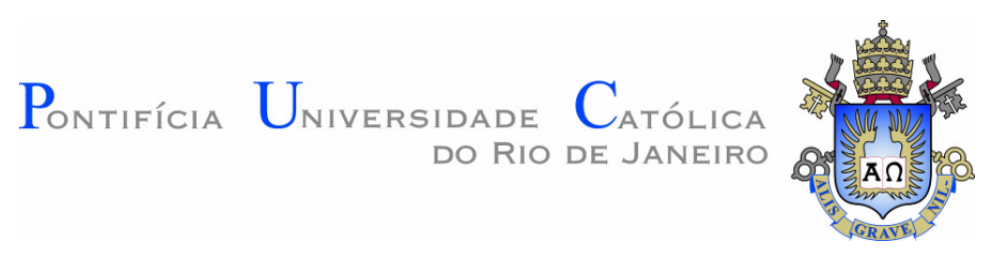

Jorge Arturo Hinostroza Medina

\title{
Avaliação de Previsões de Fratura Elastoplástica
}

\section{Tese de Doutorado}

Tese apresentada como requisito parcial para obtenção do título de Doutor pelo Programa de Pós-graduação em Engenharia Mecânica da PUC-Rio.

Orientador: Jaime Tupiassú Pinho de Castro

Rio de Janeiro

Outubro de 2014 


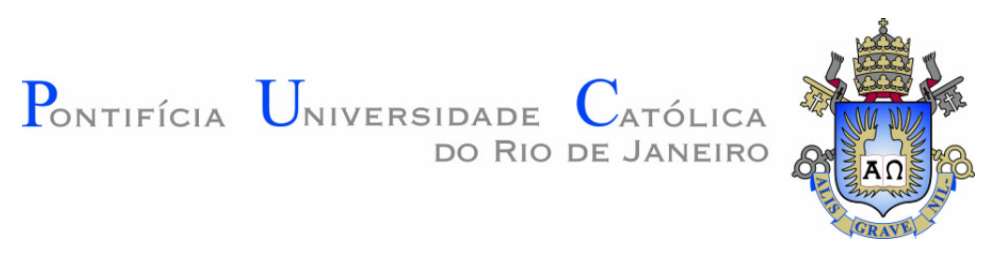

Jorge Arturo Hinostroza Medina

\title{
Avaliação de Previsões de Fratura Elastoplástica
}

\begin{abstract}
Tese apresentada como requisito parcial para obtenção do título de Doutor pelo Programa de Pós-Graduação em Engenharia Mecânica da PUC-Rio. Aprovada pela Comissão Examinadora abaixo assinada.
\end{abstract}

\author{
Prof. Jaime Tupiassú Pinho de Castro \\ Orientador \\ Departamento de Engenharia Mecânica - PUC-Rio \\ Prof. Marco Antonio Meggiolaro \\ Departamento de Engenharia Mecânica - PUC-Rio \\ Prof. Luis Fernando Martha \\ Departamento de Engenharia Civil - PUC-Rio \\ Prof. Gustavo H. Bolognesi Donato \\ Centro Universitário da FEI \\ Prof. Guilherme Peixoto Donato \\ CENPES/PETROBRAS \\ Prof. José Alexander Araújo \\ Universidade de Brasilia \\ Prof. José Eugenio Leal \\ Coordenador Setorial do Centro Técnico Científico - PUC - Rio
}


Todos os direitos reservados. É proibida a reprodução total ou parcial do trabalho sem autorização da universidade, do autor e do orientador.

\section{Jorge Arturo Hinostroza Medina}

Engenheiro Mecânico egressado da Universidad Nacional de Ingenieria (Perú), possui mestrado em Engenharia Mecânica pela Pontifícia Universidade Católica de Rio de Janeiro (PUC-Rio) com ênfase em Integridade Estrutural.

Ficha Catalográfica

Medina, Jorge Arturo Hinostroza

Avaliação de previsões de fratura elastoplástica/ Jorge Arturo Hinostroza Medina; orientador: Jaime Tupiassú Pinho de Castro. - 2014.

203 f.: il. (color); $30 \mathrm{~cm}$

Tese(doutorado) - Pontifícia Universidade Católica do Rio de Janeiro, Departamento de Engenharia Mecânica, 2014.

Inclui bibliografia

1.Engenharia mecânica-Teses. 2. Previsões elastoplásticas. 3. Fratura de materiais. 4. Tenacidade. 5. Diagrama FAD. I. Castro, Jaime Tupiassú Pinho de. II. Pontifícia Universidade Católica do Rio de Janeiro. Departamento de Engenharia Mecânica. III. Título. 


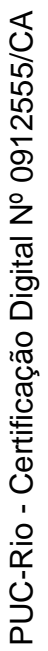

A Angel y Maria, por darme lo mejor. 


\section{Agradecimentos}

A Maru, Angel, Paty, Jakcy, Sandra, Sebas, Mica, Maca, Martin, mi Giu e todos os membros da minha querida familia por estar sempre comigo.

Ao professor Jaime Tupiassú, pela orientação e amizade durante o desenvolvimento do Doutorado.

Ao CNPq, pelo suporte financeiro.

Aos professores da PUC-Rio pelo ensino e ajuda.

Aos meus amigos do INT, especialmente a Mourad, Rodrigo, Marcelo, Juliana e Hugo.

A todos os amigos da PUC e da vida, especialmente a Gerardo, Antonio, Cristian, Marco, Rafael Goes, Jesus, Leonardo, Jaiminho, Silvia, Marco Guaman, Patty, Allan, Niurka, Edwin, Paul, Marlene, Lucia, Marko, Marco Cachito, Ronald, Juan, Maria, Lydice, Cesar,Jerson, etc,etc,etc.

A todos os funcionários do departamento de Engenharia Mecânica, pela ajuda brindada durante este tempo.

À Pontifícia Universidade Católica do Rio de Janeiro, e seus funcionários em geral. 


\section{Resumo}

Hinostroza Medina, Jorge Arturo; Pinho de Castro, Jaime Tupiassú. Avaliação de Previsões de Fratura Elastoplástica. Rio de Janeiro, 2014. 203p. Tese de Doutorado - Departamento de Engenharia Mecânica, Pontifícia Universidade Católica do Rio de Janeiro.

Neste trabalho apresenta-se uma análise dos principais procedimentos desenvolvidos para calcular a tenacidade à fratura sob condições elastoplásticas. São avaliadas as principais medidas de tenacidade, bem como as diferenças, características, fontes de conservadorismo e as principais referências bibliográficas que relatam diferenças nas metodologias de cálculo para prever as cargas de falha em estruturas com defeito. Também são analisados os principais procedimentos de avaliação da Integridade Estrutural de estruturas com defeito (Fitness For Service, FFS). Testes de tenacidade são feitos variando as condições de restrição em torno da trinca (geometria do $\mathrm{CP}$, tamanho inicial de trinca, entalhe lateral). Foram levantadas as curvas $\mathrm{J}_{\mathrm{R}}$ para todos os CPs testados, e previsões de carga de fratura são feitas segundo os principais procedimentos FFS, e testes são realizados em placas trincadas centralmente com o objetivo de registrar as cargas de falha do defeito, para compará-las com as previsões dos procedimentos FFS e avaliar o conservadorismo de cada um deles. Como outra contribuição ao trabalho, simulações numéricas dos ensaios de tenacidade são desenvolvidas com o objetivos de reproduzir os testes feitos e calibrar os parâmetros de simulação de rasgamento dúctil em estruturas com defeito.

\section{Palavras-chave}

Previsões Elastoplásticas; Fratura de Materiais;Tenacidade; Diagrama FAD. 


\section{Abstract}

Hinostroza Medina, Jorge Arturo; Pinho de Castro, Jaime Tupiassú (Advisor). Evaluation of elastoplastic fracture predictions. Rio de Janeiro, 2014. 203p. Doctoral Thesis - Departamento de Engenharia Mecânica, Pontifícia Universidade Católica do Rio de Janeiro.

This work presents an analysis of the main procedures developed to calculate fracture toughness under elastoplastic conditions. The main measures of toughness, differences, characteristics, and sources of conservatism are evaluated. Also, the main bibliographies reporting differences in calculation methods to predict failure loads in flawed structures are examined. Lastly, the main assessment procedures of Structural Integrity of structures with flaws (fitness for service, FFS) are also analyzed.Toughness tests are performed varying the constraint conditions near the crack tip (geometry, initial crack size, side grooves). $\mathrm{J}_{\mathrm{R}}$ curves and fracture load predictions were made according to the principal FFS procedures. Also, experimental tests are performed on centrally cracked plates in order to record the failure loads of the structure, to compare them with the predictions of FFS procedures and to evaluate their conservatism. Other contributions of this work are numerical simulations of toughness tests, developed in order to reproduce the tests and calibrate the simulation parameters of ductile tearing in structures with flaws.

\section{Keywords}

Elastoplastic prediction; Fracture; Toughness; FAD diagram. 


\section{Sumário}

1 . Introdução 19

1.1. Objetivos 20

1.2. Revisão bibliográfica $\quad 21$

1.3. Conteúdo da tese $\quad 24$

2. Conceitos básicos da Mecânica da Fratura 26

2.1. Mecânica da Fratura 26

2.1.1. Ductilidade 28

2.1.2. Tenacidade à Fratura 29

2.2. Mecânica da Linear Elástica (MFLE) 30

2.2.1. Fator de concentração de tensões $\left(\mathrm{K}_{\mathrm{t}}\right)$

2.2.2. Balanço de energia de Griffith 33

2.2.3. Taxa de liberação de Energia $G$

2.2.4. Fator de intensidade de tensões (K) 35

2.2.5. Zona Plástica na ponta da trinca 37

2.2.6. Restrição à deformação plástica 41

2.2.7. O Parâmetro $\mathrm{K}_{\mathrm{lc}}$

2.3. Mecânica da Fratura Elasto-Plástica (MFEP) 45

2.3.1. Deslocamento da abertura da ponta da trinca (CTOD) 46

2.3.2. O Modelo de Wells 47

2.3.3. O Modelo de Dugdale, Burdekin e Stone 48

2.3.4. O Modelo de Dawes 49

2.3.5. A Integral J 50

2.3.6. A Curva $J_{R} \quad 51$

2.4. Mecânica da Fratura Bi-Paramétrica 52

2.4.1. Tensão $T$

2.4.2. O Parâmetro Q 56

2.5. Modelo de Gurson $\quad 57$

3 . Estudo dos principais procedimentos para cálculo da Tenacidade 
3.1. Corpos de Prova (CP) 61

3.2. Pré-trinca de fadiga 63

3.3. Side Grooves (Entalhes Laterais) 65

3.4. Procedimento de ensaio $\mathrm{K}_{\mathrm{lc}} \quad 67$

3.5. Procedimento de ensaio CTOD 71

3.5.1. Comentários a respeito do cálculo de CTOD 72

3.6. Procedimento de ensaio J 74

3.7. Procedimento para obtenção da curva $J_{R} \quad 76$

3.7.1. Comentários a respeito do cálculo da curva $J_{R} \quad 81$

3.8. Procedimento para obtenção da curva JR em CPs SE(T) 82

4. Integridade Estrutural e Metodologias Fitness for Service 85

4.1. Integridade Estrutural 85

4.2. Avaliação da Integridade Estrutural (AIE) 85

4.3. Falha 85

4.4. A metodologia Fitness for Service (FFS) 85

4.4.1. Procedimentos Simplificados 87

4.4.2. Procedimentos Detalhados 88

4.4.3. Procedimentos baseados no FAD 88

4.4.4. Procedimentos baseados no CDFD 93

4.4.5. Procedimentos baseados em ambas as metodologias 96

4.4.6. Procedimento BS 7910:2005 [10] 99

5 . Testes de caracterização do material 119

5.1. Metalografia 119

5.2. Teste de tração 120

5.3. Testes de tenacidade à fratura 122

5.3.1. Corpos de Prova (CPs) 122

5.3.2. Pré-trinca de fadiga 124

5.3.3. Entalhes Laterais (Side Grooves) 125

5.3.4. Testes CPs SE(B) 126

5.3.5. Testes CPs SE(T) 131

5.3.6. Fratura dos CPs 135

$\begin{array}{ll}\text { 5.3.7. Curvas } J_{R} & 137\end{array}$ 
5.3.8. Análise Fratográfica 142

5.4. Simulações dos testes de Tenacidade 144

6. Cálculo e Validação de Previsões de Integridade Estrutural de Placas Trincadas por Vários Métodos de FFS 150

6.1. Previsões das Normas FFS 150

6.1.1. Estrutura analisada $\quad 150$

6.1.2. Avaliação Nível 1

6.1.3. Avaliação Nível 2

6.1.4. Avaliação Nível $3 \quad 158$

6.1.5. Resultados e Comparações 159

6.1.6. Comentários ao respeito das comparações 162

6.2. Testes Estruturais de Validação 163

6.2.1. Placa A12-01 167

6.2.2. Placa A08-01 168

6.2.3. Placa A08-02 169

6.2.4. Placa A10-01 169

6.2.5. Placa A12-02 170

6.2.6. Comentários a respeito dos Testes Estruturais de Validação 171

7. Considerações Finais 172

7.1. Conclusões 172

8. Referências Bibliográficas 174

$\begin{array}{ll}\text { Apêndice A } & 183\end{array}$

A.1. Superfícies de fratura dos CPs testados 183

A.2. Fratografias dos CPs testados 188

Apêndice B - Medição de deslocamento em teste de Tenacidade usando a Metodologia DIC 


\section{Lista de Figuras}

Figura 2.1 - Triangulo da Mecânica da Fratura. 27

Figura 2.2 - Limite de aplicação da MFLE[22]. 27

Figura 2.3 - Fratura Dúctil[23]. 28

Figura 2.4 - Fratura Frágil[23]. 29

Figura 2.5 - Diferentes estados de tensão[24]. 31

Figura 2.6 - Fragilização por estado triaxial de tensões[24]. 32

Figura 2.7 - A placa de Inglis[28]. 32

Figura 2.8 - Campo de tensões em torno da ponta da trinca. 35

Figura 2.9 - Tensões normais ao plano da trinca[28]. 37

Figura 2.10 - Tamanho da zona plástica de Irwin[28]. 38

Figura 2.11 - Segunda estimativa da zona plástica de Irwin[28]. $\quad 39$

Figura 2.12 - Zona plástica segundo Dugdale[28]. 40

Figura 2.13 - Modelos de correção da zona plástica[28]. 40

Figura 2.14 - Origem do efeito da restrição plástica[30].

Figura 2.15 - Condições de restrição em trincas[30]. 42

Figura 2.16 - Capacidade máxima de restrição para uma trinca[30]. 42

Figura 2.17 - Relaxação da restrição da ponta da trinca[30]. 43

Figura 2.18 - Relação Kı vs. espessura - Adaptado de [31].

Figura 2.19 - Esquema aplicação Mecânica da Fratura[24]. 46

Figura 2.20 - Abertura da ponta da trinca (CTOD). $\quad 47$

Figura 2.21 - Estimativa CTOD considerando o modelo de Irwin[28]. 48

Figura 2.22 - Estimativa CTOD-Modelo de Dugdale[33]. 48

Figura 2.23 - Comportamento do CP no teste CTOD, adaptado de [28].49

Figura 2.24 - Curvas EP,NLE e caminho s englobando a área S [22]. 51

Figura 2.25 - Curva $J_{R}$, adaptada de[28]. 52

Figura 2.26 - Curva $J_{R}$ vs. tamanho de trinca[34]. 53

Figura 2.27 - a/W vs. $\beta[28]$. $\quad 55$

Figura 2.28 - Influência Geométrica vs. Tenacidade[36]. 56

Figura 2.29 - Zona de fratura dúctil [38].

Figura 2.30 - Células computacionais (Material : Gurson-Tvergaard). 60

Figura 3.1 - CP de Flexão em três pontos - SE(B). 61 
Figura 3.2 - CP Compacto de tração - C(T). 61

Figura 3.3 - CP Compacto de tração em forma de disco DC(T) 62

Figura 3.4 - CP de tração em forma de arco - A(T) 62

Figura 3.5 - CP Compacto de tração com entalhe escalonado C(T)-EE 62

Figura 3.6 - CPs não convencionais. 64

Figura 3.7 - Pré-trinca e carregamento variável. 64

Figura 3.8 - Side Groove. $\quad 66$

Figura 3.9 - Tipos de Side Groove. $\quad 66$

Figura 3.10 - Frentes de trinca em CP SE(B), (A) sem side groove,

(B) tipo U, (C) tipo V V, (D) fenda de 0,2 mm [44]. 67

Figura 3.11 - Diferentes tipos de registro do teste 68

Figura 3.12 - Esquema para medição de ao. 69

Figura 3.13 - Esquema para cálculo de $\mathrm{PQ}_{Q} 70$

Figura 3.14 - Esquema para cálculo da tenacidade. 71

Figura 3.15 - Cálculo de Vp. $\quad 72$

Figura 3.16 - Cálculo do CTOD nas diferentes normas. 73

$\begin{array}{ll}\text { Figura } 3.17 \text { - Área Força(P) vs deslocamento do clip gage(V). } & 74\end{array}$

Figura 3.18 - Cálculo do J nas diferentes normas. 75

Figura 3.19 - Método "unloading compliance".. 76

Figura 3.20 - Curva JR [3].

Figura 3.21 - Curva JR segundo a norma ASTM E-1820[3] 78

Figura 3.22 - Região de dados qualificados da Curva JR [3].

Figura 4.1 - Procedimento J-T. 87

Figura 4.2 - FAD R6. $\quad 90$

Figura 4.3- Crack driving force diagram[52]. 95

Figura 4.4 - Stability assessment diagram[52]. 95

Figura 4.5 - FAD Nível 1.

Figura 4.6 - Tipos de defeito. 105

Figura 4.7 - Diagrama de fluxo Nível 1 [10]. 106

Figura 4.8 - Formulas para $\sigma_{\text {ref }}[10]$. 109

Figura 4.9 - FAD Nível 2A. 110

Figura 4.10 - Diagrama de fluxo Nível 2[10]. 112

Figura 4.11 - Diagrama de fluxo Nível 3 [10] . 116

Figura 4.12 -Lugar geomêtrico no Nível 3. 
Figura 5.1 - Amostras embutidas em baquelite.

Figura 5.2 - Metalografía (A) aumento de 100x (B) aumento de 200x. 120

Figura 5.3 - C.P. tração segundo a norma ASTM E 8M[56]. 120

Figura 5.4 - Máquina de tração INSTRON 8872.

Figura 5.5 - Sequência do teste. 121

Figura 5.6 - Curvas tensão vs. deformação. 121

Figura 5.7 - Dimensões do CP tipo SE(B) - dimensões em mm. 122

Figura 5.8 - Dimensões do CP tipo SE(T) - dimensões em mm. 123

Figura 5.9 - CPs usinados. 123

Figura 5.10 - Montagem do CP para pré-trincamento 124

Figura 5.11 - Detalhe da pré-trinca. 125

Figura 5.12 - Tela de acomanhamento do pré-trincado. 125

Figura 5.13 - CP com entalhe lateral. 126

Figura 5.14 - Conjunto de CPs entalhados lateralmente. 126

Figura 5.15 - Posicionamento do clip gage no CP. 127

Figura 5.16 - Curva $P$ vs. $\operatorname{COD}(a / W=0,5) . \quad 128$

Figura 5.17 - Zona plástica CPs A) SEB 05-02 e B) SEB 05-03. 128

Figura 5.18 - Curva $P$ vs. $\operatorname{COD}(a / W=0,4) . \quad 129$

Figura 5.19 - Curva P vs. COD (a/W=0,3). 130

Figura 5.20 - Curva P vs. COD totais. 130

Figura 5.21 - Curvas P vs. COD para CPs mais representativos. 131

Figura 5.22 - Propagação estável da trinca. 131

Figura 5.23 - Montagem do teste SE(T). 132

Figura 5.24- Curva $P$ vs. $\operatorname{COD}(a / W=0,5)$. 132

Figura 5.25-Zona plástica CPs A) SET 05-02 e B) SET 05-03. 133

Figura 5.26- Curva P vs. $\operatorname{COD}(a / W=0,4)$. 134

Figura 5.27-Curva P vs. COD totais. 134

Figura 5.28- Curvas P vs. COD para CPs mais representativos. 135

Figura 5.29 - Propagação estável da trinca. 135

Figura 5.30 - Curvas P vs. COD totais. 136

Figura 5.31 - CPs submersos em nitrogênio líquido para fratura. $\quad 136$

Figura 5.32 - Fratura dos CPs após resfriamento. 136

Figura 5.33 - Esquema da medição dos comprimentos das trincas. 137

Figura 5.34 - Curva JR dos CPs SE(B) a/W=0,3. 138 
Figura 5.35 - Curva $J_{R}$ dos CPs SE(B) a/W=0,4. 138

Figura 5.36 - Curva JR dos CPs SE(B) a/W=0,5. 139

Figura 5.37 - Curvas $J_{R} S E(B)$ totais. 139

Figura 5.38 - Curva $J_{R}$ dos CPs SE(T) a/W=0,4. 140

Figura 5.39 - Curva $J_{R}$ dos CPs SE(T) a/W=0,5. 140

Figura 5.40 - Curvas $J_{R} S E(T)$ totais. 141

Figura 5.41 - Curvas $J_{R}$ totais. 141

Figura 5.42 - Fratografia \# 1 da superficie CP SEB 05-02. 142

Figura 5.43 - Fratografia \# 2 da Superfície CP SEB 05-02. 142

Figura 5.44 - Fratografia \#3 da superfície do CP SEB 05-02. 143

Figura 5.45 - Fratografia \#1 da superfície do CP SET 04-03. 143

Figura 5.46 - Fratografia \#2 da superfície do CP SET 04-03. 143

Figura 5.47 - Fratografia \#3 da superfície do CP SET 04-03. 144

Figura 5.48 - Geometria do CP usado no modelo. 144

Figura 5.49 - Malha de E.F. e células computacionais no CP. 145

Figura 5.50 - Condições de contorno do modelo. 146

Figura 5.51 - Tensão de von Mises (frame 200 e 4800) - a/W=0,3. 147

Figura 5.52 - Curvas $P$ vs COD a/W=0,5 experimental e numérico. $\quad 148$

Figura 5.53 - Curvas $P$ vs COD a/W=0,4 experimental e numérico. 148

Figura 5.54 - Curvas $P$ vs COD a/W=0,3 experimental e numérico. $\quad 149$

Figura 5.55 - Curvas P vs COD ajustadas. 149

Figura 6.1 - Defeito considerado na placa. 150

Figura 6.2 - Diagrama Nível 1 (200MPa). 154

Figura 6.3 - Diagrama Nível 1. 154

Figura 6.4 - Diagrama Nível 1. 155

Figura 6.5 - Diagrama FAD Nível 2A e B. 156

Figura 6.6 - Diagrama Nível 2 (200MPa). 157

Figura 6.7 - Diagrama Nível 2A. 158

Figura 6.8 - Diagrama Nível 2B. 158

Figura 6.9 - Diagrama Nível 3.

Figura 6.10 - Diagrama Nível 3B. 159

Figura 6.11 - Usinagem das placas, dimensões em mm. 163

$\begin{array}{ll}\text { Figura } 6.12 \text { - Placas com entalhe central . } & 164\end{array}$

Figura 6.13 - Máquina e equipamentos usados no pré-trincado. 164 
Figura 6.14- Detalhe da placa entalhada.

Figura 6.15 - Pré-trinca aproximadamente semieliptica placa A12-01. 165

Figura 6.16 - Montagem do ensaio nas duas máquinas. 166

Figura 6.17 - Inicio e fim do teste - Placa A12-01. 166

Figura 6.18- Tensão vs. deformação - Placa A12-01. 167

Figura 6.19 - F.S. da Placa A12-01. 168

Figura 6.20 - F.S. da Placa A08-01. 168

Figura 6.21 - F.S. da Placa A08-02. 169

Figura 6.22 - F.S. da Placa A10-01. 170

Figura 6.23 - F.S. da Placa A12-02. 170

Figura A.1 - Superfície de fratura CP SEB-03-01. 183

Figura A.2 - Superfície de fratura CP SEB-03-02. 183

Figura A.3 - Superfície de fratura CP SEB-03-03. 183

Figura A.4 - Superfície de fratura CP SEB-04-01. 184

Figura A.5 - Superfície de fratura CP SEB-04-02. 184

Figura A.6 - Superfície de fratura CP SEB-04-03. 184

Figura A.7 - Superfície de fratura CP SEB-05-01. 185

Figura A.8 - Superfície de fratura CP SEB-05-02. 185

Figura A.9 - Superfície de fratura CP SEB-05-03. 185

Figura A.10 - Superfície de fratura CP SET-04-01. 186

Figura A.11 - Superfície de fratura CP SET-04-02. 186

Figura A.12 - Superfície de fratura CP SET-04-03. 186

Figura A.13 - Superfície de fratura CP SET-05-01. 187

Figura A.14 - Superfície de fratura CP SET-05-02. 187

Figura A.15 - Superfície de fratura CP SET-05-03. 187

Figura A.16 - Fratografias \# 1 e \# 2 da superfície CP SEB 03-01. 188

Figura A.17 - Fratografia \# 3 da superfície CP SEB 03-01. 188

Figura A.18 - Fratografias \# 1 e \# 2 da superfície CP SEB 03-02. 188

Figura A.19 - Fratografia \# 3 da superfície CP SEB 03-02. 189

Figura A.20 - Fratografias \# 1 e \# 2 da superfície CP SEB 03-03. 189

Figura A.21 - Fratografia \# 3 da superfície CP SEB 03-03. 189

Figura A.22 - Fratografias \# 1 e \# 2 da superfície CP SEB 04-01. 190

Figura A.23 - Fratografia \# 3 da superfície CP SEB 04-01. 190

Figura A.24 - Fratografias \# 1 e \# 2 da superfície CP SEB 04-02. 190 
Figura A.25 - Fratografia \# 3 da superfície CP SEB 04-02. 191

Figura A.26 - Fratografias \# 1 e \# 2 da superfície CP SEB 04-03. 191

Figura A.27 - Fratografia \# 3 da superfície CP SEB 04-03. 191

Figura A.28 - Fratografias \# 1 e \# 2 da superfície CP SEB 05-01. 192

Figura A.29 - Fratografia \# 3 da superfície CP SEB 05-01. 192

Figura A.30 - Fratografias \# 1 e \# 2 da superfície CP SEB 05-03. 192

Figura A.31 - Fratografia \# 3 da superfície CP SEB 05-03. 193

Figura A.32 - Fratografias \# 1 e \# 2 da superfície CP SET 04-01. 193

Figura A.33 - Fratografias \# 1 e \# 2 da superfície CP SET 04-02. 193

Figura A.34 - Fratografias \# 1 e \# 2 da superfície CP SET 04-03. 194

Figura A.35 - Fratografias \# 1 e \# 2 da superfície CP SET 05-01. 194

Figura A.36 - Fratografias \# 1 e \# 2 da superfície CP SET 05-02. 194

Figura A.37 - Fratografias \# 1 e \# 2 da superfície CP SET 05-03. 195

Figura B.1- Clip gage montado no CP. 197

Figura B.2- Geometria e dimensões do CP. 198

Figura B.3- Sistema de monitoramento da pré-trinca. 198

Figura B.4- Aplicação da tinta (a)CP;(b) clip gage;(c) conjunto ;

(d) padrão de pontos

Figura B.5- Sistema de monitoramento : (a) câmera CCD e lente;

(b) CP e clip gage a fotografar; (c) tela de alta resolução;

(d) processamento das imagens DIC. 200

Figura B.6- Imagens tiradas pelo software DIC para o CP3. 201

Figura B.7- Processamento de imagem CP3. 201

Figura B.8- Curva força vs.LLD - CP1. 202

Figura B.9- Curva força vs.LLD - CP2. 202

Figura B.10- Curva força vs.LLD - CP3. 203

Figura B.11- Curva força vs.LLD - CP4. 203 


\section{Lista de tabelas}

Tabela 3.1 - Tipos de CPs para cálculo de Kıc . 63

Tabela 3.2 - Tipos de CPs para cálculo de CTOD. 63

Tabela 3.3 - Tipos de CPs para cálculo de J. 63

Tabela 3.4 - Valores de ao segundo cada procedimento. 65

Tabela 4.1 - Tarefas e subtarefas do projeto[53]. 97

Tabela 4.2 - Estrutura matricial do projeto FITNET[54]. 98

Tabela 4.3 - Estrutura da BS 7910 [10]. 102

Tabela 5.1 - Propriedades mecânicas. 122

Tabela 5.1 - Nomenclatura dos CPs. 123

Tabela 5.2 - Parâmetros de teste. 127

Tabela 5.3 - Propriedades mecânicas CPs SEB. 140

Tabela 5.4 - Propriedades mecânicas CPs SET. 141

Tabela 5.5 - Número de elementos e nós nos modelos. 145

Tabela 6.1 - Dimensões dos defeitos nas placas avaliadas. 151

Tabela 6.2 - Tensões críticas no nível 1 - Placa A12-01. 160

Tabela 6.3 - Tensões críticas no nível 1.

Tabela 6.4 - Tensões críticas no nível 2A - Placa A12-01. 160

Tabela 6.5 - Tensões críticas no nível 2A. 161

Tabela 6.6 - Tensões críticas no nível 2B-Placa A12-01. 161

Tabela 6.7 - Tensões críticas no nível 2B. 161

Tabela 6.8 - Tensões críticas no nível 3A-Placa A12-01. 161

Tabela 6.9 - Tensões críticas no nível 3A. 162

Tabela 6.10 - Tensões críticas no nível 3B-Placa A12-01. 162

Tabela 6.11 - Tensões críticas no nível 3B. 162

Tabela 6.12 - Nomenclatura das placas. 163

Tabela 6.13 - Tensões de falha e F.S. previstos para a placa A12-01. 167

Tabela 6.14 - Tensões de falha e F.S. previstos para a placa A08-01. 168

Tabela 6.15 - Tensões de falha e F.S. previstos para a placa A08-02. 169

Tabela 6.16 - Tensões de falha e F.S. previstos para a placa A10-01. 169

Tabela 6.17 - Tensões de falha e F.S. previstos para a placa A12-02. 170

Tabela 6.18 - Maiores e menores previsões nas placas. 
Tabela B.1 - Taxa de carregamento e tempo dos testes. 